\title{
Stochastic evolution equations in portfolio credit modelling
}

\author{
N. Bush*, B. M. Hambly ${ }^{\dagger}$ H. Haworth ${ }^{\ddagger}$ and C. Reisinger
}

July 16, 2009

\begin{abstract}
We consider a structural credit model for a large portfolio of credit risky assets. By considering the large portfolio limit we introduce a stochastic partial differential equation which describes the evolution of the density of asset values. The loss function of the portfolio is then a function of the evolution of this density at the default boundary. We develop numerical methods for pricing and calibration of the model to credit indices and consider its performance pre and post credit crunch. We also use it to price dynamic credit products such as forward starting CDO tranches.
\end{abstract}

\section{Introduction}

The rapid growth of the credit derivatives market over the past decade has led to the development of more complex credit instruments requiring new mathematical models for pricing and risk management. The subsequent contraction due to the credit crunch has placed even more emphasis on the importance of understanding the risks involved in dealing with complex credit products.

Unfortunately credit quality, the underlying of all credit products, is not an observable in the market and there is no canonical approach to its modelling. The two natural models that have been extensively developed are the structural approach and the reduced form approach, and each has been extended to the portfolio setting in a variety of ways. In this paper our focus will be on the large portfolio limit of a multidimensional structural model. By taking this limit we obtain a stochastic partial differential equation which models the evolution of the value of a large basket of underlying assets. The key quantities for multiname credit are then certain functions of the solution of this stochastic partial differential equation.

The modelling of the individual entities in a credit basket is a bottom-up approach to the problem of pricing multiname credit products. This approach (whether structural or reduced form) is currently widely used and a reason for this has been the introduction of copulas and the subsequent factor models. Copulas are functions that link univariate marginals to their full marginal distribution. Within finance they completely separate the problem of specifying the marginal distributions from the problem of specifying the market co-movement. Thus default

\footnotetext{
${ }^{*} \mathrm{KBC}$ AIM

${ }^{\dagger}$ Mathematical Institute, University of Oxford, 24-29 St Giles, Oxford OX1 3LB, UK.

E-mail: hambly@maths.ox.ac.uk

$\ddagger_{\text {Credit Suisse }}$

E-mail: helen.haworth@linacre.oxon.org

$\S$ Mathematical Institute, University of Oxford, 24-29 St Giles, Oxford OX1 3LB, UK.

E-mail: reisinge@maths.ox.ac.uk

The authors are grateful to Credit Suisse for the market data used for the calibration.

The views expressed in this article are those of the authors and not those of Credit Suisse.
} 
information can be estimated from the single name market and the copula then maps these marginals, with a correlation structure, to the full joint default distribution.

This led to the development of conditionally independent factor (CIF) models where the existence of a low dimensional set of market factors is assumed. Individual default times become mutually independent when conditioned on these market factors, and using this independence joint default times can easily be calculated using integral transforms or recursive arguments. This led to a huge increase in computational efficiency. Coupled with its simplicity and ease of implementation these models became very popular. If Gaussian random variables are used then this has been the market's standard model.

However, as the portfolio credit market expanded it became clear that these models were unable to cope with some of the new instruments. Copula and CIF models have no dynamics to speak of; nowhere is it specified how their parameters or underlyings evolve. Furthermore, they only model expected defaults within one time period (Making copula parameters time dependent leads to prices that are not arbitrage free). For instruments such as collateralised debt obligations (CDO) this is not an issue as they are essentially one period instruments, but for those with stronger timing features this is not acceptable. These two points make it impossible to price dynamic instruments such as options on CDOs and very difficult to price multi-period instruments such as forward starting CDOs. Thus our purpose is to develop a relatively simple dynamic extension of a CIF to the large portfolio setting.

An alternative multi-asset route is a top-down approach which is a relatively recent innovation. Here the joint default distribution is modeled directly without regard to the single name market. It may seem that relevant information is being ignored, but now modellers do not need to worry about imposing a correlation structure. The correlation is an inherent property of the quantity being modeled, hence does not need to be specified. This leaves the modellers free to concentrate on how the joint distribution evolves through time, an issue frequently ignored in the bottom-up approach.

Although many of the exotic credit instruments have traded infrequently, especially post the credit crunch, their introduction highlighted the need for a more sophisticated approach to portfolio credit modelling. There is a large and rapidly growing literature in this area, so we only mention a few [6], [30], [10], [1], [26]. Using the top-down approach, frameworks similar to that of the HJM interest rate models were developed for the joint loss distribution. Other top-down approaches include the Markov chain model in [29] and the models of [2], [9] and [7]. Reducedform approaches have been extended to more than one issuer via correlated stochastic parameters. A relatively tractable example is the intensity-gamma model by [21]; another is the affine jump diffusion model of [24]. Lastly structural models, which are inherently dynamic, have been recast in a multidimensional space using correlated asset value processes. In this setting, formulae only exist in rare instances; beyond that numerical methods must be employed which can be very time consuming. [25] provides an overview of some of the main bottom-up approaches.

Our model falls into the class of multi-dimensional structural models and takes the approach of modelling the empirical measure of the asset prices in the basket when the underlyings have dynamics linked through a factor model. The pricing of CDOs is then a function of the limit of the 
empirical measure of the large basket. This limit is captured by a stochastic partial differential equation (SPDE) and requires numerical techniques for its solution. One natural approach is just to use a Monte Carlo technique to simulate the whole basket, and for small sizes of basket this would be a natural approach. However, as the basket size increases, the numerical solution of the limit SPDE becomes more computationally efficient and we discuss this in our simplified setting.

We begin with a description of the mechanics and basic valuation methods of synthetic collateralised debt obligations in Section 2 in order to provide the necessary background for later sections. In Section 3 we introduce structural models. After providing a brief survey of prior work in the area, we develop a model for portfolio credit starting from a multidimensional structural model. We make strong assumptions with the aim of delivering a relatively simple, tractable model that encapsulates the information required to calculate the loss distribution for a portfolio of risky assets. The aim in Section 4 is to develop a suitable numerical scheme for solving the SPDE. Section 5 discusses the application of the model to tranche pricing and the valuation of two different types of forward starting CDO contracts and uses the simplified model to generate some example forward spreads.

\section{Collateralised Debt Obligations}

Collateralised debt obligations (CDOs) are securitized interests in pools of credit risky assets. These assets can include mortgages, bonds, loans and credit derivatives. The CDO repackages the credit risk of the reference portfolio into multiple tranches that are then passed on to investors. Although there are many different types of CDO, here we will be focussing on what is known as a synthetic CDO i.e. one whose collateral pool consists entirely of credit default swaps.

To date, the synthetic CDO, credit indices and single name CDS market together make up the majority of the total traded notional in the credit derivative market. It is possible to trade single tranches within a synthetic CDO without the entire structure being constructed. In this case the two parties of the transaction, the protection buyer and protection seller, exchange payments as if the CDO had been set-up. The performance of this single tranche CDO (STCDO) is dependent on the number of defaults that occur in the reference portfolio during the lifetime of the contract.

Each tranche is defined by two points that determine its place within the capital structure: the attachment point and the higher valued detachment point. These are usually expressed as a percentage of the total portfolio notional. The tranche notional is defined as the difference between the attachment and detachment points. When losses are incurred, and the cumulative loss in the collateral pool is between the attachment and detachment point, the seller pays the buyer an amount equal to the loss incurred within the tranche. The tranche notional is then reduced by this amount. This means that when the cumulative loss exceeds the detachment point the tranche notional is zero. In return for this protection, the buyer pays a quarterly premium based off a fixed spread and the outstanding tranche notional.

Say we have $N$ entities in our reference credit portfolio each with notional $N_{0}$. We define the 
total loss $L_{t}$ on the portfolio as

$$
L_{t}=\sum_{i=1}^{N} L_{i} 1_{\left\{\tau_{i} \leq t\right\}},
$$

where $L_{i}=N_{0}\left(1-R_{i}\right), R_{i}$ and $\tau_{i}$ are the recovery rate and default time of the ith entity respectively. If we assume the recovery rate is the same across all credit entities and equal to a value $R$ then we can write

$$
L_{t}=N_{0}(1-R) \sum_{i=1}^{N} 1_{\left\{\tau_{i} \leq t\right\}} .
$$

The outstanding tranche notional, $Z_{t}$, of a single tranche within a synthetic CDO is given by

$$
Z_{t}=\left[d-L_{t}\right]^{+}-\left[a-L_{t}\right]^{+}
$$

and the tranche loss $Y_{t}$ as

$$
Y_{t}=\left[L_{t}-a\right]^{+}-\left[L_{t}-d\right]^{+}
$$

where $a$ is the tranche attachment point and $d$ is the tranche detachment point.

As for a Credit Default Swap (CDS) the value of a STCDO is given by the difference between the fee leg and the protection leg. The protection buyer pays a regular fixed spread on the outstanding notional of the tranche. We denote the payment dates by $T_{i}, 1 \leq i \leq n$, the intervals by $\delta_{i}=T_{i}=T_{i-1}$ and the value of a bank account at time $t$ by $b(t)$. Then the value of the fee leg is given by

$$
s V^{f e e}=s \sum_{i=1}^{n} \frac{\delta_{i}}{b\left(T_{i}\right)} \mathbb{E}^{\mathbb{Q}}\left[Z_{T_{i}}\right] .
$$

The protection seller only makes payments to the buyer when the tranche incurs losses, and the value of this payment is equal to the change in the tranche loss $Y_{t}$. However, we can express the value of the protection leg in terms of the outstanding tranche notional $X_{t}$ as follows

$$
V^{\text {prot }}=\sum_{i=1}^{n} \frac{1}{b\left(T_{i}\right)} \mathbb{E}^{\mathbb{Q}}\left[Z_{T_{i-1}}-Z_{T_{i}}\right]
$$

As in a CDS contract the par spread $s$ of the tranche is chosen to make the initial value zero hence is calculated as

$$
s=\frac{V^{\text {prot }}}{V^{f e e}} .
$$

From (2.5) and (2.6) we see that the key to finding the par spread is obtaining the distribution of the outstanding tranche notional; from (2.3), this is equivalent to finding the distribution of the loss $L_{t}$. As all portfolio credit derivatives are essentially options on this loss variable the heart of every multiname credit model is determining its distribution.

\section{Structural Models}

Structural models are based on the premise that when a company's asset value falls below a certain threshold barrier a default is triggered. The first model of this type was introduced by [23] and considers a company whose debt comprises a single zero coupon bond and whose assets 
follow a geometric Brownian motion. If on the redemption date of the bond the value of the assets is not sufficient to cover the face value of the debt then the company is deemed to have defaulted.

In Merton's model default can only happen at a single moment in time which could be seen as slightly unrealistic. [3] used a different approach that utilised the theory of first exit times to construct a model that allows default at any moment. Their model assumes default occurs at the first time the asset value reaches a certain threshold level. To date, there are many variants of this model but the basic type is as follows. Let $A_{t}$ be the company's asset value whose process is governed by

$$
\frac{d A_{t}}{A_{t}}=\mu d t+\sigma d W_{t}
$$

where $\mu$ is the mean rate of return on the assets, $\sigma$ is the asset volatility and $W_{t}$ is a standard Brownian motion. If we denote the default threshold barrier by $B_{t}$ we define the distance to default, $X_{t}$, as

$$
X_{t}=\frac{1}{\sigma}\left(\log A_{t}-\log B_{t}\right) .
$$

The probability of default is now given by the first time $X_{t}$ hits 0 .

Structural models are appealing due to their intuitive economic interpretation and the link they provide between the equity and credit markets. They introduce spread dynamics and allow market participants to hedge spread risk with the underlying equity of the reference entity. Defaults are endogenously generated within the model and recovery rates do not need to be determined until after a default occurs.

There are however downsides that affect the practical applicability of structural models. Due to the diffusive nature of the asset process, and the assumption of perfect information regarding asset values and default thresholds, any credit event generated by the model is predictable. The immediate consequence is short term credit spreads that are near zero: a fact contradicted by empirical evidence. Extensions that try to address this issue involve increasing the near term uncertainty surrounding default. One way of doing this would be to relax the perfect information assumption, for example by making the default threshold stochastic like CreditGrades ${ }^{T M}$ described in [11], or by letting the exact value of the assets be unknown as in [8]. Another way would be to alter the diffusive properties of the asset process. [32, 33] uses a jump diffusion process and others use a more general Lévy variety e.g. [16] and [4]. As structural models are extended in these ways their analytic complexity increases dramatically. Credit spread prices can then no longer be expressed in closed form and numerical methods must be employed for pricing.

Another downside is that calibration of the model parameters is not a straightforward exercise. Debt levels are used as a proxy for the default threshold, and these can be estimated from balance sheet data and other publicly available information. Furthermore, the asset value volatility is usually inferred from the equity markets; this is assuming of course that the equity is traded. Despite these setbacks, structural models are still very popular and continue to be refined and developed. 


\subsection{Multidimensional Structural Models}

Due to the popularity enjoyed by CIF and copula models, multidimensional structural models have typically received less attention; as a result, the literature on this subject is sparse. The flaws in the standard models for the portfolio credit market have became ever more apparent leading to renewed interest in developing dynamic portfolio credit models, and those based on the firm's value were, and still are, a viable option.

The first authors to incorporate default correlation into first passage models were [34] and [17]. The former extended the Black-Cox framework to include correlated asset value processes, with hitting times being calculated from a time dependent barrier. Within the context of a portfolio containing two risky assets, closed-form expressions were derived for the joint default distribution. However, it was difficult to extend these results to more than two issuers. [17] followed Zhou's approach and moved to a higher dimensional space but had to sacrifice the analytic results. In their setup, defaults are only allowed to occur at discrete time intervals and a piecewise constant default barrier is calibrated to match implied default rates. The authors used Monte-Carlo simulation to obtain prices.

In [18] the asset value processes for a multi-dimensional structural model are correlated via a set of common factors. Again, piecewise default barriers are calibrated to match market prices and Monte-Carlo simulation is used to value STCDOs. A comparison is performed between this setup and the single factor Gaussian copula. Then the model is extended to use either stochastic recovery rates or a stochastic correlation.

[12] extend a first passage model with stochastic volatility to multiple names. Co-dependence is introduced via correlated driving Brownian motions in the asset processes and also through common stochastic volatility factors. They derive a PDE for the joint survival probability of the portfolio and using asymptotic expansions they investigate the loss distribution of a basket of defaultable bonds.

In a more recent paper, [15] consider default contagion within a structural model framework. Default contagion tries to capture the notion of an asymmetric default correlation between companies. They model this idea by assuming that the default of one company causes the the volatility of the other companies' asset processes to jump. The magnitude of this effect is then related to the correlation between the companies. Theoretical results are provided for $n$ dimensions but numerical limitations restrict simulations to portfolios containing two or three assets.

[6] and [5] study interacting particle systems based on hidden Markov chains and perform a numerical analysis of importance sampling versus interacting particle systems respectively.

\subsection{The Structural Evolution Model}

The starting point for our model is very similar to that used in [18]. We begin by considering a portfolio that references the credit default swaps of $N$ different companies. Denoting the companies' asset values at time $t$ by $A_{t}^{i}$ we assume that under the risk neutral measure they follow a 
diffusion process given by

$$
d A_{t}^{i}=r A_{t}^{i} d t+\sigma_{i}\left(t, A_{t}^{i}\right) d W_{t}^{i}+\sum_{j=1}^{m} \sigma_{i j}\left(t, A_{t}^{i}\right) d M_{t}^{j},
$$

where the $W_{t}^{i}$ and $M_{t}^{j}$ are Brownian motions satisfying

$$
d\left[W_{t}^{i}, M_{t}^{j}\right]=0 \quad \forall i, j
$$

and

$$
d\left[W_{t}^{i}, W_{t}^{j}\right]=d\left[M_{t}^{i}, M_{t}^{j}\right]=\delta_{i j} d t,
$$

where we have written [., .] for the quadratic covariation and will use [.] for the quadratic variation. In order to develop a simple model we make some simplifying assumptions. Firstly we assume that $m=1$ and secondly that $\sigma_{i}^{2}=\rho \sigma^{2}, \sigma_{i 1}^{2}=(1-\rho) \sigma^{2}$, where $\sigma^{2}$ is constant and $\rho \in[0,1]$ is the constant correlation. Thus we can write (3.2) in terms of the distance to default process $X_{t}=\left(\ln A_{t}-\ln B\right) / \sigma$, with constant barrier $B$, as

$$
d X_{t}^{i}=\mu d t+\sqrt{\rho} d M_{t}+\sqrt{1-\rho} d W_{t}^{i},
$$

where $\mu=\left(r-\frac{1}{2} \sigma^{2}\right) / \sigma$. Note the co-dependence between the asset processes is provided solely by the Brownian motion $M_{t}$ which can be thought of as a market wide factor influencing all of the assets.

We will approximate the system by a measure valued process. In this paper we will just outline the derivation of the equation in this simple setting, a full proof in a more general setting than that of (3.2) can be found in [14]. Let $\nu_{N, t}$ denote the equally weighted empirical measure for the entire portfolio given by

$$
\nu_{N, t}=\frac{1}{N} \sum_{i=1}^{N} \delta_{X_{t}^{i}},
$$

where $\delta_{x}$ is a Dirac measure at the point $x$. Let $\phi \in C_{c}^{\infty}(\mathbb{R})$, the space of infinitely differentiable continuous functions with compact support, and for a measure $\nu_{t}$ we write

$$
\left\langle\phi, \nu_{t}\right\rangle=\int \phi(x) \nu_{t}(d x)
$$

Using the empirical measure (3.3) we define a family of processes $F_{t}^{N, \phi}$ by

$$
F_{t}^{N, \phi}=\left\langle\phi, \nu_{N, t}\right\rangle=\frac{1}{N} \sum_{i=1}^{N} \phi\left(X_{t}^{i}\right)
$$

Applying Ito's formula to $F_{t}^{N, \phi}$ we have

$$
\begin{aligned}
F_{t}^{N, \phi}= & F_{0}^{N, \phi}+\int_{0}^{t} \frac{1}{N} \sum_{i=1}^{N}\left(\phi^{\prime}\left(X_{s}^{i}\right) d X_{s}^{i}+\frac{1}{2} \phi^{\prime \prime}\left(X_{s}^{i}\right) d\left[X_{s}^{i}\right]\right) \\
= & \frac{1}{N} \sum_{i=1}^{N} \int_{0}^{t} \phi^{\prime}\left(X_{s}^{i}\right)\left[\mu d s+\sqrt{1-\rho} d W_{s}^{i}+\sqrt{\rho} d M_{s}\right] \\
& +\frac{1}{N} \sum_{i=1}^{N} \int_{0}^{t} \frac{1}{2} \phi^{\prime \prime}\left(X_{s}^{i}\right) d s .
\end{aligned}
$$


If we define the second order linear operator $\mathcal{A}$ by

$$
\mathcal{A}=\mu \frac{\partial}{\partial x}+\frac{1}{2} \frac{\partial^{2}}{\partial x^{2}}
$$

we can rewrite (3.6) in terms of (3.4) as

$$
F_{t}^{N, \phi}=F_{0}^{N, \phi}+\int_{0}^{t}\left\langle\mathcal{A} \phi, \nu_{N, s}\right\rangle d s+\int_{0}^{t}\left\langle\sqrt{\rho} \phi^{\prime}, \nu_{N, s}\right\rangle d M_{s}+\int_{0}^{t} \frac{1}{N} \sum_{i=1}^{N} \phi^{\prime}\left(X_{s}^{i}\right) \sqrt{1-\rho} d W_{s}^{i}
$$

We now pass to the limit by letting $N \rightarrow \infty$. In order to determine what happens we focus on the idiosyncratic term in (3.7) i.e.

$$
I_{t, N}^{\phi}=\int_{0}^{t} \frac{1}{N} \sum_{i=1}^{N} \sqrt{1-\rho} \phi^{\prime}\left(X_{s}^{i}\right) d W_{s}^{i} .
$$

By the independence of the $W_{t}^{i}$ this is a martingale with quadratic variation equal to

$$
\left[I_{N}^{\phi}\right]_{t}=\int_{0}^{t} \frac{1}{N^{2}} \sum_{i=1}^{N}(1-\rho)\left(\phi^{\prime}\left(X_{s}^{i}\right)\right)^{2} d s .
$$

It is not difficult to show that there exists a constant $K_{\phi, t}$ such that $\mathbb{P}$-almost surely

$$
\lim _{N \rightarrow \infty} \frac{1}{N} \sum_{i=1}^{N} \int_{0}^{t}(1-\rho)\left|\phi^{\prime}\left(X_{s}^{i}\right)\right|^{2} d s \leq K_{\phi, t}
$$

and hence, with $\mathbb{P}$ probability 1 , we have

$$
\lim _{N \rightarrow \infty} \frac{1}{N^{2}} \sum_{i=1}^{N} \int_{0}^{t}(1-\rho)\left|\phi^{\prime}\left(X_{s}^{i}\right)\right|^{2} d s \leq \lim _{N \rightarrow \infty} \frac{1}{N} K_{\phi, t}=0 .
$$

Thus the random term due to the idiosyncratic component of the asset values has become deterministic in the infinite dimensional limit and must vanish. As the market factors affect each company to the same degree, we can write

$$
F_{t}^{N, \phi} \rightarrow F_{t}^{\phi}=\left\langle\phi, \nu_{t}\right\rangle \quad \text { as } N \rightarrow \infty,
$$

with

$$
d F_{t}^{\phi}=\left\langle\mathcal{A} \phi, \nu_{t}\right\rangle d t+\left\langle\sqrt{\rho} \phi^{\prime}, \nu_{t}\right\rangle d M_{t}
$$

Alternatively, we can write this in an integrated form as

$$
\left\langle\phi, \nu_{t}\right\rangle=\left\langle\phi, \nu_{0}\right\rangle+\int_{0}^{t}\left\langle\mathcal{A} \phi, \nu_{s}\right\rangle d s+\int_{0}^{t}\left\langle\sqrt{\rho} \phi^{\prime}, \nu_{s}\right\rangle d M_{s}
$$

Equation (3.9) describes the evolution of the empirical measure in the weak sense. Having derived this particular representation for the problem we would now like to recharacterise it in terms of a stochastic PDE. In order to do this we need the measure $\nu_{t}$ to be absolutely continuous with respect to the Lebesgue measure in order to write $\nu_{t}(d x)=v(t, x) d x$ for some density $v$ (for the 
full proof see [14]). Substituting the Lebesgue representation for the empirical measure into (3.9), integrating by parts and writing $\mathcal{A}^{\dagger}$ for the adjoint operator of $\mathcal{A}$, we get

$$
\begin{aligned}
\int \phi(x) v(t, x) d x= & \int \phi(x) v(0, x) d x+\int_{0}^{t} \int \mathcal{A} \phi(x) v(s, x) d x d s \\
& \quad+\int_{0}^{t} \int \sqrt{\rho} \phi^{\prime}(x) v(s, x) d x d M_{s} \\
= & \int \phi(x) v(0, x) d x+\int_{0}^{t} \int \phi(x) \mathcal{A}^{\dagger} v(s, x) d x d s \\
& \quad-\int_{0}^{t} \int \phi(x) \frac{\partial}{\partial x}(\sqrt{\rho} v(s, x)) d x d M_{s} \\
= & \int \phi(x)\left(v(0, x)+\int_{0}^{t} \mathcal{A}^{\dagger} v(s, x) d s-\int_{0}^{t} \frac{\partial}{\partial x}(\sqrt{\rho} v(s, x)) d M_{s}\right) d x .
\end{aligned}
$$

As this holds $\forall \phi \in C_{c}^{\infty}(\mathbb{R})$ we must have

$$
v(t, x)=v(0, x)+\int_{0}^{t} \mathcal{A}^{\dagger} v(s, x) d s-\int_{0}^{t} \frac{\partial}{\partial x}(\sqrt{\rho} v(s, x)) d M_{s} .
$$

Alternatively, we can write this in differential form and by expanding the adjoint of the operator $\mathcal{A}$ we have

$$
d v(t, x)=-\mu \frac{\partial v}{\partial x}(t, x) d t+\frac{1}{2} \frac{\partial^{2} v}{\partial x^{2}}(t, x) d t-\sqrt{\rho} \frac{\partial v}{\partial x}(t, x) \mathrm{d} M_{t} .
$$

This is a stochastic PDE that describes the evolution of the distance to default of an infinite portfolio of assets whose dynamics are given by (3.2).

We now wish to incorporate a default condition into the model. For this we suppose that an asset defaults if its distance to default is 0 and in this case the asset is removed from the portfolio which implies that the density of the assets at the barrier is 0 . We introduce this into our model with the Dirichlet boundary condition

$$
v(t, 0)=0 \quad \text { for } t \geq 0,
$$

which corresponds to absorption at the barrier.

We can now use the limiting empirical measure $\nu_{t}$ to approximate the loss distribution for a portfolio of fixed size $N$ whose assets also follow (3.2). We do this by matching the initial conditions, thus setting

$$
v(0, x)=\frac{1}{N} \sum_{i=1}^{N} \delta_{X_{0}^{i}}(x),
$$

where the $X_{0}^{i}>0, i=1, \ldots, N$ are the initial values for the distance to default of the assets in our fixed portfolio of size $N$.

\subsection{The Portfolio Loss}

We would like to price portfolio credit derivatives whose values depend on the cumulative defaults occurring within a reference basket of risky assets. The key to pricing these instruments is determining the joint loss distribution. We have just derived an equation that describes the 
evolution of the empirical measure of the limiting large portfolio of assets. At any future value in time, we can determine the loss in the portfolio by calculating the total mass of the empirical measure of assets that have not defaulted. Thus the portfolio loss $L_{t}^{N}$ can be approximated by

$$
L_{t}^{N}=N L_{t}
$$

where

$$
L_{t}=1-\int_{0+}^{\infty} v(t, x) d x
$$

and $N$ is the number of assets in the portfolio. We note that given the initial condition (3.13) we have

$$
L_{0}^{N}=N\left(1-\frac{1}{N} \sum_{i=1}^{N} \int_{0+}^{\infty} \delta_{X_{0}^{i}}(x) d x\right)=0 .
$$

Also, due to the way in which defaults are incorporated into the model we have

$$
\begin{array}{cc}
0 \leq L_{t} \leq 1, & \text { for } t \geq 0 \\
P\left(L_{s} \geq K\right) \leq P\left(L_{t} \geq K\right), & \text { for } s \leq t,
\end{array}
$$

which ensures that there is no arbitrage in the loss distribution. Both of these properties are expected for a model of cumulative loss in a portfolio.

We summarize the SPDE for the limit of the system of SDE's as

$$
\left\{\begin{array}{l}
d v=-\frac{1}{\sigma}\left(r-\frac{1}{2} \sigma^{2}\right) v_{x} d t+\frac{1}{2} v_{x x} d t-\sqrt{\rho} v_{x} d M(t), \\
v(0, x)=v_{0}(x), \quad v(t, 0)=0 .
\end{array}\right.
$$

\subsection{Solving the SPDE}

The SPDE (3.14) without the boundary condition is easily solved as

$$
v(t, x)=u\left(t, x-\sqrt{\rho} M_{t}\right), \quad \forall x \in \mathbb{R}, t>0,
$$

where $u(t, x)$ is the solution to the deterministic PDE

$$
u_{t}=\frac{1}{2}(1-\rho) u_{x x}-\frac{1}{\sigma}\left(r-\frac{1}{2} \sigma^{2}\right) u_{x},
$$

with $u(0, x)=v_{0}(x)$.

The SPDE with the boundary condition has been treated in [22]. This allows us to state an existence and uniqueness theorem. Firstly we give some notation. Let $(\Omega, \mathcal{F}, \mathbb{P})$ be a probability space supporting a one-dimensional Brownian motion $\left(M_{t}, \mathcal{F}_{t}\right)$. Let $\mathcal{P}$ denote the $\sigma$-algebra of predictable sets on $\Omega \times \mathbb{R}_{+}$associated with the filtration $\mathcal{F}_{t}$ and $H^{1}(D)=\left\{f: f \in L_{2}(D), f^{\prime} \in\right.$ $\left.L_{2}(D)\right\}$, where $L_{2}(D)=\left\{f: \int_{D} f^{2} d x<\infty\right\}$, for a set $D \subset \mathbb{R}$ is the usual Sobolev space.

Theorem 3.1 The SPDE (3.14) has a unique solution $u \in L_{2}\left(\Omega \times(0, T), \mathcal{P}, H^{1}((0, \infty))\right)$ and is such that $x u_{x x} \in L_{2}\left(\Omega \times(0, T), \mathcal{P}, L_{2}((0, \infty))\right)$. 
Proof: The result follows from Theorem 2.1 of [22]. Thus all we have to do is ensure that the conditions of that Theorem hold in our setting. The boundary of the domain $\mathbb{R}_{+}$is the single point 0 and this satisfies the Hypothesis 2.1 of [22]. The coefficients of our SPDE are constants and hence trivially satisfy Hypotheses 2.2 and 2.3, with the function $\psi(x)=\min (x, 1)$, which satisfies the required assumptions in [22], and Hypothesis 2.4.

\subsection{A Connection with Filtering}

We note that the SPDE can be viewed as a PDE with a Brownian drift. This is easily seen through an interpretation as the Zakai equation for a filtering problem. Let $(\tilde{\Omega}, \tilde{\mathcal{F}}, \tilde{\mathbb{P}})$ be a probability space. Under $\tilde{\mathbb{P}}$ we define the signal process $X$ to be a stochastic process satisfying

$$
\begin{aligned}
d X & =\mu d t-\sqrt{\rho} d M+d W, \quad t \leq \tau_{0} \\
X_{t} & =0, t>\tau_{0}
\end{aligned}
$$

where $\tau_{0}=\inf \left\{t: X_{t}=0\right\}$, where $\mu, \rho$ are constants and $M$ and $W$ are independent Brownian motions and $X_{0}=x$. The observation process $Y$ is taken to be just the market noise,

$$
d Y_{t}=d M_{t}
$$

then the Zakai equation for the probability density of the signal given the observations is exactly our SPDE.

Thus, by standard filtering theory, if we want to compute a functional of the signal we need to calculate

$$
m_{\psi}(t)=\tilde{\mathbb{E}}\left(\psi\left(X_{t}\right) \mid \mathcal{F}_{t}^{M}\right)=\int \psi(y) u_{t}(y) d y
$$

This means that the probability distribution for the position of a company given the market noise is the density $u(t, x)$ satisfying

$$
d u(t, x)=\left(-\mu u_{x}(t, x)+\frac{1}{2} u_{x x}(t, x)\right) d t-\sqrt{\rho} u_{x}(t, x) d M_{t},
$$

with $u(0, x)=u_{0}(x)$, that is the initial guess at $X_{0}$ is the density $u_{0}(x)$ and $u(t, 0)=0$. Thus for the loss function we are interested in computing the proportion of companies that have defaulted by time $t$ and this can be found by computing $m_{\psi}(t)$ for $\psi(t)=I_{\left\{\tau_{0}<t\right\}}$. If we start from a given fixed point so that $u_{0}(x)$ is a delta function at $x$. Then

$$
L_{t}=m_{\psi}(t)=\tilde{\mathbb{P}}^{x}\left(\inf _{s \leq t} X_{s}<0 \mid \mathcal{F}_{t}^{M}\right) .
$$

Now the process $X$ can be written as a Brownian motion with drift

$$
X_{t}=x+\mu t-\sqrt{\rho} M_{t}+W_{t}
$$

and if we are given $M$, this can be expressed as

$$
X_{t}=x+f(t)+W_{t},
$$


where $f(t)=\mu t-\sqrt{\rho} M_{t}$ is a deterministic time dependent drift function which is a fixed random path.

Thus to compute the random loss function we need

$$
\begin{aligned}
\tilde{\mathbb{P}}^{x}\left(\inf _{s \leq t} X_{s}<0 \mid \mathcal{F}_{t}^{M}\right) & =\tilde{\mathbb{P}}^{0}\left(\inf _{s \leq t} x+f(s)+W_{s}<0 \mid \mathcal{F}_{t}^{M}\right) \\
& =\tilde{\mathbb{P}}\left(\inf _{s \leq t} f_{s}+W_{s}<-x \mid \mathcal{F}_{t}^{M}\right) .
\end{aligned}
$$

The case where we have a general initial distribution $u_{0}(x)$ is then

$$
L_{t}=\int_{0}^{\infty} u_{0}(x) \tilde{\mathbb{P}}\left(\inf _{s \leq t} f_{s}+W_{s}<-x \mid \mathcal{F}_{t}^{M}\right) d x .
$$

Thus we can try to compute this by solving the hitting time problem for Brownian motion with time dependent drift for a fixed realization of the market noise. It is straightforward to use this to simulate a realization of the loss function.

To derive this SPDE we made some simplifying assumptions. The first of these arose when specifying the asset processes in (3.2). We had to set the drift and volatility of all the assets to some common value. For the drift this is not a problem, because under the risk neutral measure it will be transformed to a value that excludes arbitrage. The fact that there is only one yield curve means that this value will be the same for all assets. If our reference portfolio contained entities denominated in more than one currency this would not be the case and some approximation would have to be made.

This argument cannot be used for the volatility as it is not affected by a change of measure. Therefore, it would seem that giving the assets one common value of volatility is a very restrictive assumption. However, for any given value of the volatility we still have the freedom to choose the default barrier specific to any one asset. Via the distance-to-default transformation this freedom manifests itself in our particular choice of starting value for each process. The effect of changing the barrier and changing the volatility is very similar. To see this note that default risk is measured by how many standard deviations away from the barrier our process is. To increase the default risk we need to reduce this distance which can be done by either increasing the standard deviation or moving the barrier closer. Although these are clearly not equivalent transformations they have a very similar effect and so the single volatility assumption is not as restrictive as it initially appears.

Having a single volatility number also eases calibration as we do not have to estimate the volatilities of all of the entities within our portfolio. Instead, we will have to replace it by some 'average' market volatility. Not only will this help day-to-day calibration stability but it means that credit derivative prices will be a function of one volatility parameter only. This is usually a desirable property from a practitioner's point of view as it allows one to take a view on that parameter; this cannot be done if there were a single parameter for each entity within our portfolio.

The major simplification that allowed us to derive our SPDE came when we moved to an infinite dimensional limit. In this limit, the idiosyncratic noise of the individual assets is averaged out. In fact, we could have any number of idiosyncratic components, provided they are independent and uncorrelated, and they would average out to zero. It is only the correlated components 
between the assets that remain i.e. the market risk. Note that this means that if the limiting portfolio was fully diversified, that is had no correlation, there would be no noise in the limit and the limit portfolio would evolve deterministically!

\section{Numerical Methods}

Our starting point is the SPDE (3.14). The overall structure of the simulation algorithm requires the numerical solution of a PDE for each simulated path of the market factor. From the PDE solution, quantities like tranche losses can be computed, which are then averaged over the simulated paths. A Monte Carlo method on top of a PDE solver is computationally costly. In the presence of non-smooth initial data, and with senior tranche prices depending on very low probability events, a carefully adapted numerical scheme is necessary.

\subsection{Setup}

Deviating slightly from the absorbing boundary condition in (3.14), we make the assumption that defaults are only observed at a discrete set of times, which we take quarterly to coincide with the payment dates. If a firm's value is below the default barrier on one of the observation dates $T_{i}$, it is considered defaulted and removed from the basket. We therefore solve the modified SPDE problem

$$
\begin{array}{rc}
d v=-\frac{1}{\sigma}\left(r-\frac{1}{2} \sigma^{2}\right) v_{x} d t+\frac{1}{2} v_{x x} d t-\sqrt{\rho} v_{x} d M(t) & t \in\left(T_{k}, T_{k+1}\right), 0 \leq k<n \\
v(0, x)=v_{0}(x) & \\
v\left(T_{k}, x\right)=0 & \forall x \leq 0,0<k \leq n
\end{array}
$$

We can take advantage of this computationally as the boundary condition is not active in intervals $\left(T_{k}, T_{k+1}\right)$, and therefore the Brownian driver only introduces a normally distributed shift to the solution, accumulated over the entire interval. Using the solution given in (3.15) it can therefore be written as

$$
v(t, x)=\left\{\begin{array}{rl}
0 & x \leq 0 \wedge t \in\left\{T_{k}, 1 \leq k \leq n\right\} \\
v^{(k)}\left(t-T_{k}, x-\sqrt{\rho}\left(M(t)-M\left(T_{k}\right)\right)\right) & \text { else if } t \in\left(T_{k}, T_{k+1}\right], 0 \leq k<n
\end{array}\right.
$$

where $v^{(k)}$ is the solution to the (deterministic) problem

$$
\begin{aligned}
v_{t}^{(k)} & =\frac{1}{2}(1-\rho) v_{x x}^{(k)}-\frac{1}{\sigma}\left(r-\frac{1}{2} \sigma^{2}\right) v_{x}^{(k)}, \quad t \in(0, \tau)=\left(0, T_{k+1}-T_{k}\right) \\
v^{(k)}(0, x) & =v\left(T_{k}, x\right)
\end{aligned}
$$

assuming payment dates are equally spaced with intervals $\tau=T_{k+1}-T_{k}$.

This suggests the following inductive strategy for $k=0, \ldots, n-1$ :

1. Start with $v^{(0)}(0, x)=v_{0}(x)$.

2. Solve the PDE (4.5) numerically in the interval $\left(0, T_{1}\right)$, to obtain $v^{(0)}\left(T_{1}, x\right)$. 
3. Simulate $M\left(T_{1}\right)$, evaluate $v\left(T_{1}, x\right)$ according to (4.4).

4. For $k>0$, having computed $v\left(T_{k}, x\right)$ in the previous step, use this as initial condition for $v^{(k)}$, and repeat until $k=n$.

\subsection{Finite Differences}

To solve the PDEs (4.5) by a finite difference method, we approximate the distribution by one with support $\left[x_{\min }, x_{\max }\right]$, and set asymptotic boundary conditions $v\left(t, x_{\min }\right)=v\left(t, x_{\max }\right)=0$. Since the initial distribution is assumed localised (as it approximates the distribution of a finite number of firms), the localisation error for a given path $M$ can be made as small as needed by a suitable choice of $x_{\min }<0$ and $x_{\max }>0$. We can moreover ensure that the expected error of this approximation is much smaller than the standard error of the Monte Carlo estimates.

Then, introduce a grid $x_{0}=x_{\text {min }}, x_{1}=x_{\text {min }}+\Delta x, \ldots, x_{\text {min }}+j \Delta x, \ldots, x_{J}=x_{m i n}+J \Delta x=$ $x_{\max }$, where $\Delta x=\left(x_{\max }-x_{\min }\right) / J$, timesteps $t_{0}=0, t_{1}=\Delta t, \ldots, t_{I}=I \Delta t=\tau$, where $\Delta t=\tau / I$.

Now define an approximation $v_{j}^{i}$ to $v\left(t_{i}, x_{j}\right)$ as a solution to the difference scheme

$$
\begin{aligned}
\frac{v_{j}^{i}-v_{j}^{i-1}}{\Delta t}= & \theta\left\{\frac{1}{2} \frac{v_{j+1}^{i}-2 v_{j}^{i}+v_{j-1}^{i}}{\Delta x^{2}}-\frac{1}{\sigma}\left(r-\sigma^{2} / 2\right) \frac{v_{j+1}^{i}-v_{j-1}^{i}}{2 \Delta x}\right\} \\
& +(1-\theta)\left\{\frac{1}{2} \frac{v_{j+1}^{i-1}-2 v_{j}^{i-1}+v_{j-1}^{i-1}}{\Delta x^{2}}-\frac{1}{\sigma}\left(r-\sigma^{2} / 2\right) \frac{v_{j+1}^{i-1}-v_{j-1}^{i-1}}{2 \Delta x}\right\} .
\end{aligned}
$$

For an introduction to finite differences in financial instrument pricing see e. g. [31]. The scheme is second order accurate in $\Delta x$. The backward Euler scheme $\theta=1$ is first order accurate in $\Delta t$ and strongly $A$-stable. The Crank-Nicolson scheme $\theta=\frac{1}{2}$ is second order accurate, and is unconditionally stable in the $l_{2}$-norm for sufficiently smooth solutions, but gives rise to instabilities for initial conditions comprising $\delta$-distributions such as arise in the present setting. We address this, together with aspects of approximating non-smooth initial and interface conditions (4.3) accurately on the grid, in the following section.

\subsection{Boundary Conditions}

\subsubsection{Approximation of Boundary Condition at $t=0$}

We have initial conditions of the form

$$
v(0, x)=\frac{1}{N} \sum_{i=1}^{N} \delta\left(x-x^{i}\right),
$$

where $x^{i}$ is the initial distance to default of firm $i$. These will subsequently be implied from the firms' CDS spreads.

Numerically, it is relevant how the sum of $\delta$-distributions is best approximated on the grid. A simple approach could bin the firms into symmetric intervals of width $\Delta x$ around grid points, 
and assign the fraction of firms in such an interval to the corresponding grid value, that is

$$
v_{j}^{0}=\frac{1}{N \Delta x} \int_{x_{j}-\Delta x / 2}^{x_{j}+\Delta x / 2} \delta\left(x^{i}-x\right) \mathrm{d} x
$$

where the factor $\Delta x^{-1}$ ensures the right scaling of the density.

This approximation cannot distinguish between initial positions $x^{i}$ in an interval of length $\Delta x$, which reduces the overall order of the finite difference scheme to 1 .

To achieve higher (i. e. second) order, the $\delta$ 's have to be split between adjacent grid points. The correct weighting for a single firm with distance-to-default $x^{i}$ in the interval $\left[x_{j}, x_{j+1}\right)$, is

$$
v_{k}^{0}= \begin{cases}\Delta x^{-2}\left(x_{j+1}-x^{i}\right) & k=j \\ \Delta x^{-2}\left(x^{i}-x_{j}\right) & k=j+1 \\ 0 & \text { else }\end{cases}
$$

The extension to more firms is obvious. This can be written more elegantly as an $L_{2}$-projection of the initial condition onto the basis of 'hat functions' (linear splines) $\left\langle\Phi_{k}\right\rangle_{0 \leq k \leq N}$ where

$$
\Phi_{k}(x)=\frac{1}{\Delta x} \min \left(\max \left(x-x_{k}+\Delta x\right), \max \left(x_{k}-x, 0\right)\right),
$$

as

$$
v_{k}^{0}=\left\langle\Phi_{k}, v_{0}\right\rangle=\int_{x_{\min }}^{x_{\max }} \Phi_{k}(x) v_{0}(x) \mathrm{d} x
$$

See also [27] for applications of this idea to option pricing and estimation of sensitivities.

Note that $\Delta x \sum_{k=0}^{N} v_{k}^{0}=1$.

\subsubsection{Rannacher timestepping}

It is well-known that Crank-Nicolson timestepping has reduced convergence order for discontinuous initial conditions, and is unstable for Dirac initial conditions if the timesteps are not chosen small enough. This severely restricts the possible stepsizes and slows down the performance.

A simple fix is to replace the first Crank-Nicolson steps with backward Euler steps, a practice known as Rannacher timestepping, see [28]. The analysis in [13] shows that the optimal balance between accuracy and stability is achieved by replacing the first two Crank-Nicolson steps by four backward Euler steps of half the stepsize. We do this at $t=0$, and also at $t=T_{k}$ where the interface conditions introduce discontinuities at $x=0$.

\subsubsection{Interface conditions at $t=T_{k}$}

At $t=T_{k}$ in (4.3) we have to evaluate the grid function at shifted arguments $x_{j}-\sqrt{\rho}(M(t)-$ $\left.M\left(T_{k}\right)\right)$ that do not normally coincide with grid points. First, define a piecewise linear interpolant from the approximation $v_{j}^{I}$ obtained in the last step over the previous interval $\left[T_{k-1}, T_{k}\right]$, call it $v_{j}^{(k), I}$, as

$$
v_{\Delta x}\left(T_{k}, x\right)=\sum_{j=0}^{N} \Phi_{j}(x-\Delta M) v_{j}^{(k), I} .
$$


with $\Delta M=M\left(T_{k}\right)-M\left(T_{k-1}\right)$. Then, approximate $(4.4)$ by setting

$$
v_{j}^{(k+1), 0}=\int_{\max \left(x_{j}-\Delta x / 2,0\right)}^{x_{j}+\Delta x / 2} v_{\Delta x}\left(T_{k}, x\right) \mathrm{d} x,
$$

and use this as initial condition for the next interval (the integral is understood to be 0 if the lower limit is larger than the upper limit). This ensures that

$$
\Delta x \sum_{j=0}^{J} v_{j}^{(k+1), 0}=\int_{0}^{x_{\max }} v_{\Delta x}\left(T_{k}, x\right) \mathrm{d} x
$$

so the cumulative density of firms with firm values greater than 0 is preserved. It also has the effect that the solution is smoothed at $x=0$. This restores second order convergence. See [27] for averaging procedures to restore (higher order) convergence for non-smooth payoffs in option pricing.

\subsection{Simulations}

For a given realisation of the market factor, we can approximate the loss functional $L_{T_{k}}$ at time $T_{k}$ by

$$
L_{T_{k}}^{\Delta x}=1-\int_{0}^{x_{\max }} v_{\Delta x}\left(T_{k}, x\right) \mathrm{d} x
$$

If we explicitly include the dependence on the Monte Carlo samples $\Phi=\left(\Phi_{i}\right)_{1 \leq i \leq n}$ in $L_{T_{k}}^{\Delta x}(\Phi)$, where $\Phi_{i}$ are drawn independently from a standard normal distribution, then for $N_{\text {sims }}$ simulations with samples $\Phi^{l}=\left(\Phi_{k}^{l}\right)_{1 \leq k \leq n}, 1 \leq l \leq N_{\text {sims }}$,

$$
\begin{aligned}
\mathbb{E}^{\mathbb{Q}}\left[X_{T_{k}}\right] & \approx \mathbb{E}^{\mathbb{Q}}\left[\max \left(d-L_{T_{k}}^{\Delta x}, 0\right)-\max \left(a-L_{T_{k}}^{\Delta x}, 0\right)\right] \\
& \approx \frac{1}{N_{\text {sims }}} \sum_{l=1}^{N_{\text {sims }}}\left(\max \left(d-L_{T_{k}}^{\Delta x}\left(\Phi^{l}\right), 0\right)-\max \left(a-L_{T_{k}}^{\Delta x}\left(\Phi^{l}\right), 0\right)\right)
\end{aligned}
$$

The simulation error has two components, the discretisation error and the variance of the Monte Carlo estimate.

For the following simulations we have used these data: $T=5, r=0.027, \sigma=0.24, R=0.4$, $\rho=0.13$. Initial positions for individual firms were calibrated to their individual CDS spreads, and were well within the range $\left[x_{\min }, x_{\max }\right]=[-10,20]$.

\subsubsection{Bias}

First consider the discretisation error in $\Delta t$ and $\Delta x$, also called bias in the Monte Carlo context. We fix the path in (4.9). Fig. 1 shows how many grid points $(I)$ and how many timesteps between payment dates $(J)$ are necessary for a desired accuracy $\epsilon$. We clearly see second order convergence in $\Delta t$ and $\Delta x$. Note that the time smoothing scheme, projection of initial positions, and adapted averaging, are essential to achieve this. 

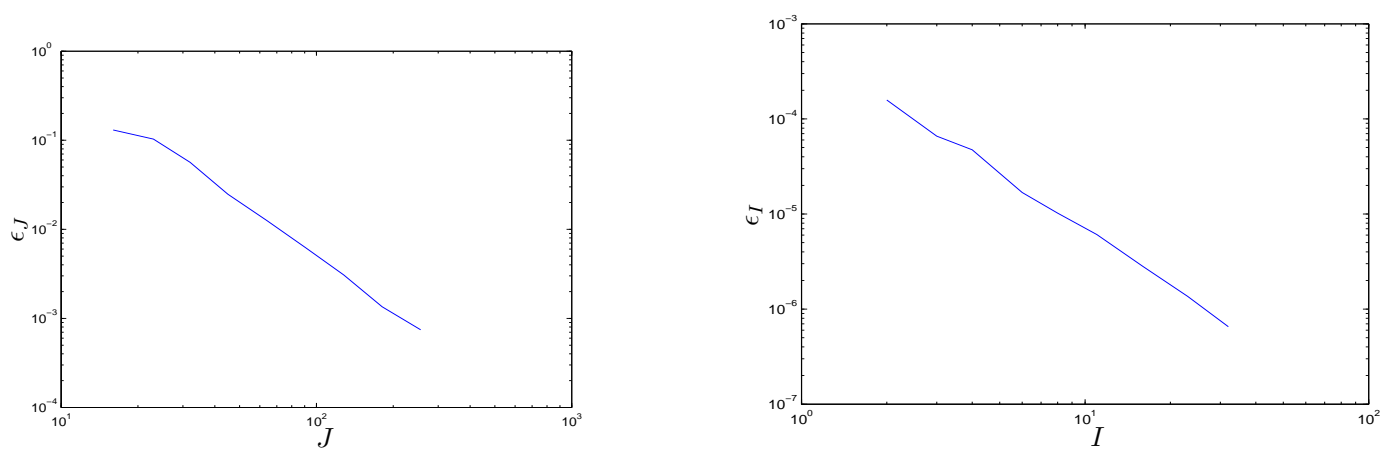

Figure 1: Extrapolation-based estimator for the discretisation error of $L_{T_{n}}$ for increasing $J$ (left) and $I$ (right) for a single realisation of the path.

\begin{tabular}{l|llllll}
$s$ & {$[0,3 \%]$} & {$[3 \%, 6 \%]$} & {$[6 \%, 9 \%]$} & {$[9 \%, 12 \%]$} & {$[12 \%, 22 \%]$} & {$[22 \%, 100 \%]$} \\
\hline & & & & & & \\
1 & $6.2725682 \mathrm{e}-03$ & $2.0969122 \mathrm{e}-02$ & $2.6111038 \mathrm{e}-02$ & $2.9513197 \mathrm{e}-02$ & $1.0000000 \mathrm{e}-01$ & $7.8000000 \mathrm{e}-01$ \\
2 & $7.9104658 \mathrm{e}-03$ & $2.2701963 \mathrm{e}-02$ & $2.7342294 \mathrm{e}-02$ & $2.9584213 \mathrm{e}-02$ & $9.9858664 \mathrm{e}-02$ & $7.8000000 \mathrm{e}-01$ \\
3 & $7.3859912 \mathrm{e}-03$ & $2.2685495 \mathrm{e}-02$ & $2.8222167 \mathrm{e}-02$ & $2.9756172 \mathrm{e}-02$ & $9.9960775 \mathrm{e}-02$ & $7.8000000 \mathrm{e}-01$ \\
4 & $7.6036670 \mathrm{e}-03$ & $2.2834542 \mathrm{e}-02$ & $2.8031717 \mathrm{e}-02$ & $2.9555681 \mathrm{e}-02$ & $9.9870460 \mathrm{e}-02$ & $7.8000000 \mathrm{e}-01$ \\
5 & $7.5088509 \mathrm{e}-03$ & $2.2772474 \mathrm{e}-02$ & $2.8037086 \mathrm{e}-02$ & $2.9510371 \mathrm{e}-02$ & $9.9844166 \mathrm{e}-02$ & $7.7999902 \mathrm{e}-01$ \\
6 & $7.4316779 \mathrm{e}-03$ & $2.2754261 \mathrm{e}-02$ & $2.8054171 \mathrm{e}-02$ & $2.9487882 \mathrm{e}-02$ & $9.9823128 \mathrm{e}-02$ & $7.7999919 \mathrm{e}-01$ \\
7 & $7.3909987 \mathrm{e}-03$ & $2.2683723 \mathrm{e}-02$ & $2.8056862 \mathrm{e}-02$ & $2.9493869 \mathrm{e}-02$ & $9.9825675 \mathrm{e}-02$ & $7.7999904 \mathrm{e}-01$ \\
8 & $7.4092772 \mathrm{e}-03$ & $2.2681457 \mathrm{e}-02$ & $2.8046172 \mathrm{e}-02$ & $2.9490874 \mathrm{e}-02$ & $9.9828340 \mathrm{e}-02$ & $7.7999870 \mathrm{e}-01$ \\
9 & $7.4051597 \mathrm{e}-03$ & $2.2676594 \mathrm{e}-02$ & $2.8052021 \mathrm{e}-02$ & $2.9493417 \mathrm{e}-02$ & $9.9828096 \mathrm{e}-02$ & $7.7999860 \mathrm{e}-01$ \\
10 & $7.4068341 \mathrm{e}-03$ & $2.2678343 \mathrm{e}-02$ & $2.8052676 \mathrm{e}-02$ & $2.9493836 \mathrm{e}-02$ & $9.9828061 \mathrm{e}-02$ & $7.7999866 \mathrm{e}-01$ \\
\hline
\end{tabular}

Table 1: Monte Carlo estimators for expected outstanding tranche notionals for $N_{\text {sims }}=64 * 4^{s-1}$ Monte Carlo runs, $s=1, \ldots, 10$. The finite difference parameters were fixed at $J=256, I=4$.

\subsubsection{Variance}

We now turn to the convergence of the Monte Carlo estimators. Table 1 shows the estimates for outstanding notionals in all tranches for an increasing number of sample paths. As the number of samples is quadrupled from one line to the next, the standard error is roughly halved as one would expect.

Fig. 2 shows better the relative error for the tranche losses for the equity, senior mezzanine, and senior tranches. The accuracy is very satisfactory for most tranches.

Defaults in the senior tranche are obviously a very unlikely event (with a probability of order $10^{-4}$ in this model), and therefore the variance is high. The computational accuracy could be improved by importance sampling techniques. However, it seems more useful to address the root of the problem first and to adapt the model to shift more weight into senior tranches, e.g. by the introduction of jumps. This is the subject of ongoing research. 

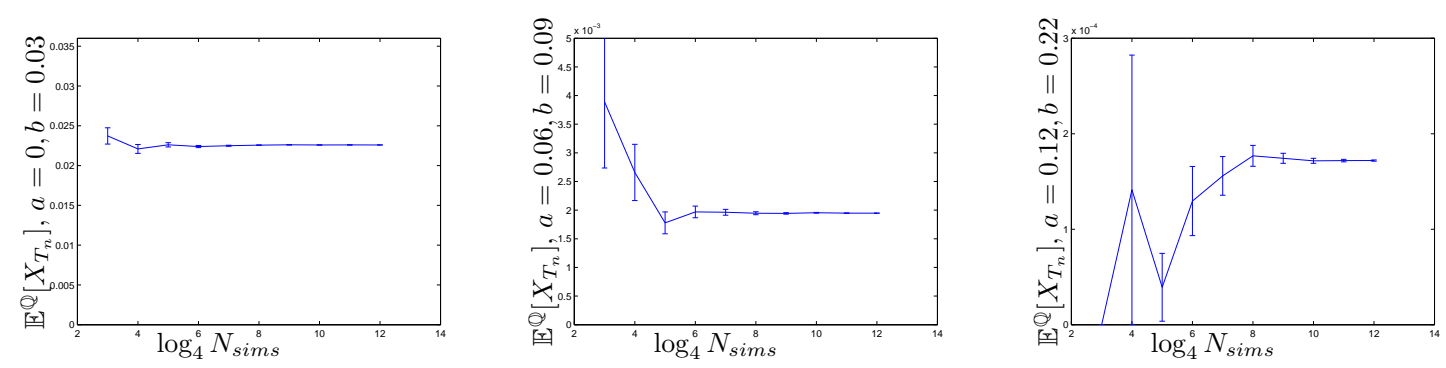

Figure 2: Monte Carlo estimators with standard error bars for expected losses in tranches $[0,3 \%]$, $[6 \%, 9 \%],[12 \%, 22 \%]$, for $N_{\text {sims }}=16 \cdot 4^{k-1}, k=1, \ldots, 10, J=256, I=4$.

\section{$5 \quad$ Market Pricing Examples}

The lack of dynamics in the market's standard pricing methodology provides the motivation for our development of a structural evolution model. This absence of dynamics made pricing some of the newer structured credit instruments very difficult and credit market developments since mid-2007 have further exposed the limitations of existing approaches, reaffirming the need for a new generation of models to enable a better understanding of the risks inherent in some of the more complex products. Many of these innovations require a coherent approach for modelling spread and/or default dynamics, for example:

- The existence of 5, 7 and 10-year index and bespoke tranches requires a model that can fit the entire correlation skew term structure, not just the correlation skew for a given time horizon.

- Forward-starting tranches require the ability to distinguish between defaults occurring before and after the forward start date.

- Options on tranches need to incorporate dynamics so that spread changes, reflecting changes in the market view of default expectations, can be captured.

- STCDOs with trigger features - for example unwind or deleveraging triggers. Some of these require full knowledge of tranche spread evolution in addition to default-loss evolution.

By investigating the behaviour of our simplified model, we are able to gain an insight into which aspects of dynamic models are important for the pricing of more exotic structured credit products. This information can then be used to help guide future model development.

We begin by analysing our model's ability to price regular index tranches for all maturities and investigate the implied correlation skew. We consider performance pre and post the onset of the credit crunch, illustrating the model's inherent ability to cope with a variety of credit environments. We then use the model to price forward index tranches.

Throughout the analysis, we infer the initial condition from market spreads for the underlying index constituents, rather than allowing it to be a free parameter to be fixed by calibration to the CDO. This is to be consistent with CDS spreads for the individual constituents. We do this by 


\begin{tabular}{|c|c|c|c|}
\hline Maturity Date & Fixed Coupon (bp) & Traded Spread (bp) & Model Spread (bp) \\
\hline $20 / 12 / 2011$ & 30 & 21 & 19.6 \\
$20 / 12 / 2013$ & 40 & 30 & 30.7 \\
$20 / 12 / 2016$ & 50 & 41 & 41.0 \\
\hline
\end{tabular}

Table 2: The fixed coupons, traded spreads and model spreads for the iTraxx Main Series 6 index on February 22, 2007. Parameters used for the model spreads are $r=0.042, \sigma=0.22, R=0.4$.

backing out the distance-to-default for each constituent from its five-year CDS spread and then aggregate these as outlined in section 4.3.1. As a consequence, the initial condition is driven by both the level of constituent spreads and their dispersion.

\subsection{Tranche Pricing}

We look first at the ability of our model to price index tranches on two dates: February 22, 2007 and December 5, 2008. We set $R=40 \%$, the level typically assumed by the market for investment grade names, and for each date, calibrate the model to 5, 7 and 10-year index spreads using the volatility, $\sigma . \quad r$ is the risk-free rate obtained from the Euro swap curve. (N.B. the correlation parameter, $\rho$, does not come into this calibration since index spreads are correlationindependent.)

Table 2 shows the traded and model index spreads for Feb 22, 2007. Since we derive the initial condition from constituent spreads, we only have one free parameter, the volatility $\sigma$, for calibrating all three index spreads. Increasing $\sigma$ to increase model spreads also causes the initial distance-to-default for each constituent to increase (since CDS spreads are fixed), so index and tranche spreads are less sensitive to changes in volatility than they would be if the initial condition was specified independently.

Table 4 shows the same results for Dec 5, 2008. In this highly distressed state, we notice that spreads are dramatically wider and the curve is inverted with 5-year $>7$-year $>10$-year spreads. Our simple model again does a good job of calibrating all three spreads. This is achieved by a smaller distance-to-default of the initial positions in combination with a lower volatility, triggering more defaults in the imminent future (see also the left plots in figures 4 and 5 later). The 5 -year point is a little low, which is a drawback of using a purely diffusive driving process: it can be hard to generate sufficient short-term losses.

For the parameters from the calibration in Table 2, Table 3 illustrates the correlation sensitivity of the 5, 7 and 10-year index tranches in the pre-crunch environment. We note that model spreads illustrate the behaviour we would anticipate:

- Equity tranche spreads decline with increasing correlation whilst spreads for other tranches generally increase with correlation. As correlation increases, there are less likely to be a few defaults, and so the equity tranche becomes less risky and its spread decreases. The probability of a greater number of defaults increases with increasing correlation and so spreads on the more senior tranches increase with correlation. 
- The exception is the 10-year junior mezzanine tranche $(3 \%-6 \%)$ which behaves more like an equity tranche and has declining spreads with increasing correlation. This is because, for the parameters used, the expected index loss is between $3 \%$ and $6 \%$. The risk of this tranche therefore decreases, along with the spread, as correlation increases, making losses in this tranche less likely.

- For the 5 and 7-year junior mezzanine and 10-year senior mezzanine tranches, spreads decline with increasing correlation for high values of correlation.

Figure 3: Implied Correlation Skew for iTraxx Main Series 6 Tranches, Feb 22, 2007.

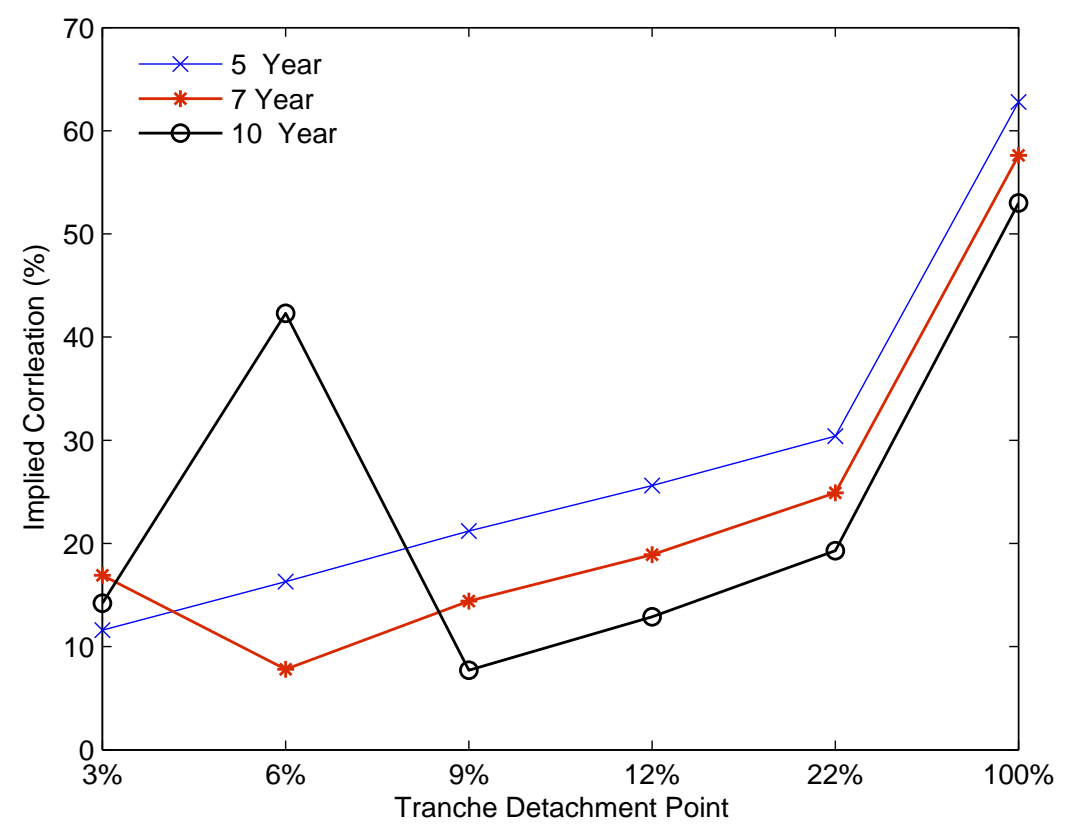

The implied correlation for each tranche is the value of correlation that gives a model tranche spread equal to the market tranche spread given in Table 3. Model parameters are $r=0.042, \sigma=0.22, R=0.4$.

Figure 3 illustrates the 5, 7 and 10-year implied correlation skew - the value of correlation that gives a model spread equal to the market spread for each tranche and maturity.

- With the exception of the 3\% - 6\% tranche, we see similar behaviour and levels for all three maturities. This consistency across the term-structure is extremely positive since it suggests that the dynamics underlying the model are realistic, even in its simple form.

- For implied correlations to be the same for all maturities across the seniority structure, 5year implied correlations generally need to decrease relative to the others and 10-year values need to increase. As mentioned before, this arises from having a purely diffusive driving process, making it hard for enough defaults to occur for short terms-to-maturity; this issue could be addressed by using a more general Levy process for the market factor. 


\begin{tabular}{|c|c|c|c|c|c|c|c|c|}
\hline & 5 Year & & & & & & & \\
\hline Tranche & Market & $\rho=0.1$ & $\rho=0.2$ & $\rho=0.3$ & $\rho=0.4$ & $\rho=0.5$ & $\rho=0.6$ & $\rho=0.7$ \\
\hline $0 \%-3 \%$ & $7.19 \%$ & $7.55 \%$ & $4.99 \%$ & $2.14 \%$ & $-0.71 \%$ & $-3.48 \%$ & $-6.17 \%$ & $-8.78 \%$ \\
\hline $3 \%-6 \%$ & 41 & 15.6 & 55.6 & 86.4 & 106.1 & 116.2 & 119.5 & 117.4 \\
\hline $6 \%-9 \%$ & 10.8 & 0.7 & 9.1 & 25 & 40.3 & 54.5 & 65.2 & 71.7 \\
\hline $9 \%-12 \%$ & 5 & 0 & 2.2 & 8.2 & 18.8 & 28.6 & 37.2 & 45.4 \\
\hline $12 \%-22 \%$ & 1.8 & 0 & 0.2 & 1.7 & 4.9 & 9.8 & 16.1 & 22.5 \\
\hline \multirow[t]{2}{*}{$22 \%-100 \%$} & 0.9 & 0 & 0 & 0 & 0.1 & 0.3 & 0.7 & 1.5 \\
\hline & 7 Year & & & & & & & \\
\hline Tranche & Market & $\rho=0.1$ & $\rho=0.2$ & $\rho=0.3$ & $\rho=0.4$ & $\rho=0.5$ & $\rho=0.6$ & $\rho=0.7$ \\
\hline $0 \%-3 \%$ & $22.1 \%$ & $27.45 \%$ & $19.97 \%$ & $13.79 \%$ & $8.31 \%$ & $3.27 \%$ & $-1.47 \%$ & $-6.04 \%$ \\
\hline $3 \%-6 \%$ & 110 & 130.6 & 183.3 & 202.2 & 206 & 201.5 & 191.6 & 177.8 \\
\hline $6 \%-9 \%$ & 32.5 & 15.3 & 52.4 & 80.5 & 99.1 & 110.6 & 116.1 & 116.9 \\
\hline $9 \%-12 \%$ & 15 & 1.8 & 17.4 & 37.1 & 54.3 & 67.1 & 76.5 & 82.7 \\
\hline $12 \%-22 \%$ & 4.9 & 0.1 & 2.3 & 8.9 & 19 & 29.9 & 39.5 & 47.9 \\
\hline \multirow[t]{2}{*}{$22 \%-100 \%$} & 2 & 0 & 0 & 0.1 & 0.4 & 1.1 & 2.3 & 4.1 \\
\hline & 10 Year & & & & & & & \\
\hline Tranche & Market & $\rho=0.1$ & $\rho=0.2$ & $\rho=0.3$ & $\rho=0.4$ & $\rho=0.5$ & $\rho=0.6$ & $\rho=0.7$ \\
\hline $0 \%-3 \%$ & $38 \%$ & $42.51 \%$ & $32.51 \%$ & $24.13 \%$ & $16.65 \%$ & $9.71 \%$ & $3.11 \%$ & $-3.33 \%$ \\
\hline $3 \%-6 \%$ & 302.5 & 375.8 & 354.9 & 331.9 & 308.1 & 283.5 & 258.3 & 231.9 \\
\hline $6 \%-9 \%$ & 83 & 101.4 & 147.3 & 166.2 & 173.6 & 174.4 & 170.8 & 163.8 \\
\hline $9 \%-12 \%$ & 37 & 24.3 & 64.1 & 90.9 & 107.7 & 117.8 & 122.9 & 124.1 \\
\hline $12 \%-22 \%$ & 12.5 & 2 & 13.5 & 29.1 & 44.4 & 57.5 & 68.2 & 76.5 \\
\hline $22 \%-100 \%$ & 3.6 & 0 & 0.1 & 0.6 & 1.5 & 3 & 5.1 & 7.7 \\
\hline
\end{tabular}

Table 3: Model tranche spreads (bp) for varying values of the correlation parameter. The equity tranches are quoted as an upfront assuming a 500bp running spread. The model is calibrated to the iTraxx Main Series 6 index for Feb 22, 2007. Market levels shown are for this date; model parameters are $r=0.042, \sigma=0.22, R=0.4$. 


\begin{tabular}{|c|c|c|c|}
\hline Maturity Date & Fixed Coupon (bp) & Traded Spread (bp) & Model Spread (bp) \\
\hline $20 / 12 / 2013$ & 120 & 215 & 207 \\
$20 / 12 / 2015$ & 125 & 195 & 195 \\
$20 / 12 / 2018$ & 130 & 175 & 176 \\
\hline
\end{tabular}

Table 4: The fixed coupons, traded spreads and model spreads for the iTraxx Main Series 10 index on December 5, 2008. Parameters used for the model spreads are $r=0.033, \sigma=0.136$, $R=0.4$.

- The behaviour of the 3\%-6\% tranche looks very different for the 7 and 10-year maturities, but actually results from the same effect: this tranche was traditionally particularly attractive to investors since its spread was perceived high for its rating, increasing demand and driving down spreads. Since 7-year tranche spreads increase with correlation, the impact of the extra demand is to decrease the implied correlation. The 10-year tranche on the other hand behaves more like an equity tranche as explained above, and spreads decrease with correlation, so the extra demand drives down spreads and therefore increases the implied correlation to give the curve shapes shown.

Table 5 shows the correlation sensitivity of the Dec 5, 2008 index tranches with parameters from the calibration in Table 4. We notice that relative to Table 3, spreads are highly distressed, the index is inverted and tranche spreads are flat to inverted across maturities. As a result, the tranches exhibit very different sensitivity to correlation than before, however there are some common themes and extensions to earlier behaviour:

- Default probabilities for the index and its constituents are very high. The index expected loss is therefore much greater than before, illustrated by the fact the first three 5-year tranches and the first four 7 and 10-year tranches have declining spreads with increasing correlation. This contrasts with just the equity and 10-year junior mezzanine tranches in Feb 2007.

- Much higher levels of $\rho$ are needed to replicate market prices than in pre-crunch times, consistent with the fact that systematic risk is a much greater concern at this time.

- Too much of our model's portfolio loss distribution lies in the middle tranches: $6 \%-22 \%$; more weight needs to be in the tail to be able to replicate $22 \%-100 \%$ tranche values. The same model shortcoming holds for all maturities, so it is not an issue with the model's term structure dynamics, which continue to exhibit realistic behaviour, but rather it reflects the need for a more sophisticated driving process.

\subsection{Forward Starting CDO Contracts}

These contracts are obligations to buy or sell protection on a specified tranche for a specified spread at some specified time in the future. Although these instruments are traded infrequently, 


\begin{tabular}{|c|c|c|c|c|c|c|c|c|}
\hline & 5 Year & & & & & & & \\
\hline Tranche & Market & $\rho=0.3$ & $\rho=0.4$ & $\rho=0.5$ & $\rho=0.6$ & $\rho=0.7$ & $\rho=0.8$ & $\rho=0.9$ \\
\hline $0 \%-3 \%$ & $71.5 \%$ & $81.88 \%$ & $75.9 \%$ & $69.56 \%$ & $63.02 \%$ & $56.25 \%$ & $49.16 \%$ & $41.65 \%$ \\
\hline $3 \%-6 \%$ & 1576.3 & 2275.2 & 1978.5 & 1743.2 & 1546.8 & 1374.6 & 1222.8 & 1090.1 \\
\hline $6 \%-9 \%$ & 811.5 & 1273.1 & 1168.2 & 1079.7 & 1001.4 & 931.3 & 864.6 & 796.3 \\
\hline $9 \%-12 \%$ & 506.1 & 775.7 & 765.8 & 748.6 & 724.7 & 695.8 & 663.2 & 629.1 \\
\hline $12 \%-22 \%$ & 180.3 & 307.8 & 353.3 & 384.7 & 405.5 & 418.1 & 423.4 & 420.5 \\
\hline \multirow[t]{2}{*}{$22 \%-100 \%$} & 77.9 & 9.2 & 16.5 & 25 & 34.3 & 44.5 & 55.7 & 68.1 \\
\hline & 7 Year & & & & & & & \\
\hline Tranche & Market & $\rho=0.3$ & $\rho=0.4$ & $\rho=0.5$ & $\rho=0.6$ & $\rho=0.7$ & $\rho=0.8$ & $\rho=0.9$ \\
\hline $0 \%-3 \%$ & $72.9 \%$ & $84.03 \%$ & $78.98 \%$ & $73.26 \%$ & $66.93 \%$ & $60 \%$ & $52.41 \%$ & $44.13 \%$ \\
\hline $3 \%-6 \%$ & 1473.2 & 2327.3 & 1985.7 & 1715.2 & 1493.4 & 1308 & 1147.8 & 1001.3 \\
\hline $6 \%-9 \%$ & 804.2 & 1344.2 & 1199 & 1085.2 & 988.2 & 900.7 & 820.9 & 747.9 \\
\hline $9 \%-12 \%$ & 512.4 & 855.4 & 808.4 & 765.3 & 725.3 & 684.8 & 643 & 600.4 \\
\hline $12 \%-22 \%$ & 182.6 & 375.4 & 401.7 & 417.6 & 425.6 & 427.4 & 423.1 & 411.8 \\
\hline \multirow[t]{2}{*}{$22 \%-100 \%$} & 75.8 & 14 & 22 & 30.6 & 39.6 & 49.3 & 59.7 & 71.2 \\
\hline & 10 Year & & & & & & & \\
\hline Tranche & Market & $\rho=0.3$ & $\rho=0.4$ & $\rho=0.5$ & $\rho=0.6$ & $\rho=0.7$ & $\rho=0.8$ & $\rho=0.9$ \\
\hline $0 \%-3 \%$ & $73.8 \%$ & $85.13 \%$ & $80.57 \%$ & $74.99 \%$ & $68.51 \%$ & $61.31 \%$ & $53.31 \%$ & $44.22 \%$ \\
\hline $3 \%-6 \%$ & 1385.5 & 2270.8 & 1895.7 & 1611.1 & 1385.8 & 1195.3 & 1032 & 889.6 \\
\hline $6 \%-9 \%$ & 824.7 & 1332.2 & 1164.2 & 1033.7 & 925.5 & 833.5 & 749.8 & 669.7 \\
\hline $9 \%-12 \%$ & 526.1 & 870.8 & 798.8 & 740.7 & 689.3 & 640.5 & 592.1 & 543.1 \\
\hline $12 \%-22 \%$ & 174.1 & 406.1 & 414.9 & 417.5 & 415.6 & 409.8 & 400.2 & 385.3 \\
\hline $22 \%-100 \%$ & 76.3 & 18.3 & 26.1 & 34 & 42.1 & 50.6 & 59.7 & 69.8 \\
\hline
\end{tabular}

Table 5: Model tranche spreads (bp) for varying values of the correlation parameter. The equity tranches are quoted as an upfront assuming a 500bp running spread. The model is calibrated to the iTraxx Main Series 10 index for Dec 5, 2008. Market levels shown are for this date; model parameters are $r=0.033, \sigma=0.136, R=0.4$. 
their pricing and hedging is an active research topic. We will look at two types of forward starting CDO: one that resets the cumulative loss at the forward start date and one that does not. For discussion purposes we will refer to these as resetting and non-resetting respectively but it should be borne in mind that these are not standard market terms.

\subsubsection{Non-Resetting Forward CDO Tranche}

For a non-resetting forward CDO tranche defined over the time interval $\left[T, T^{*}\right]$, the cumulative losses incurred up to time $T$ count towards the total loss in the tranche for all $t$ with $T<t$. This feature makes pricing straightforward and analogous to a forward CDS contract.

Consider a portfolio with $m$ entities in the reference portfolio. We define the total loss on the portfolio at time $t$ by

$$
L_{t}=\sum_{i=1}^{m} L_{i} 1_{\left\{\tau_{i} \leq t\right\}} .
$$

If the forward tranche has attachment point $a$ and detachment point $d$ then the outstanding tranche notional, $Z_{t}$, is given as

$$
Z_{t}=\left[d-L_{t}\right]^{+}-\left[a-L_{t}\right]^{+}
$$

The value of the forward tranche contract is again given by the difference between the fee leg and the protection leg. So far the setup has been the same as the standard CDO tranche. The only difference when pricing this forward contract is the fact that now we are only interested in the payment dates $T_{i}, i=1, \ldots, n$ where $T<T_{1}<\ldots,<T_{n} \leq T^{*}$. Using these payment dates the present value of the coupon payments given a forward spread $s$ is

$$
s V^{f e e}=s \sum_{i=1}^{n} \frac{\delta_{i}}{b\left(T_{i}\right)} \mathbb{E}^{\mathbb{Q}}\left[Z_{T_{i}}\right]
$$

The protection leg is given by

$$
V^{\text {prot }}=\sum_{i=1}^{n} \frac{1}{b\left(T_{i}\right)} \mathbb{E}^{\mathbb{Q}}\left[Z_{T_{i-1}}-Z_{T_{i}}\right] .
$$

Today, the value of the forward starting contract is zero and hence the forward break-even spread is given by

$$
s=\frac{V^{\text {prot }}}{V^{f e e}} .
$$

\subsubsection{Resetting Forward CDO Tranche}

With this contract, the cumulative loss up to time $T$ is ignored and the value of the tranche is dependent only on the further loss incurred after time $T$. If the forward tranche has attachment $a$ and detachment $d$ then this is equivalent to a non-resetting forward tranche with attachment $\left(L_{T}+a\right)$ and detachment $\left(L_{T}+d\right)$. Using the same payment dates as the non-resetting forward contract we define the effective forward loss at time $T_{i}$ by

$$
\hat{L}_{T_{i}}=L_{T_{i}}-L_{T}
$$


which gives the forward tranche notional as

$$
Z_{t}=\left[d-\hat{L}_{t}\right]^{+}-\left[a-\hat{L}_{t}\right]^{+} .
$$

With these new definitions the forward break-even spread can be calculated as before using (5.3), (5.4) and (5.5).

\subsection{Forward Pricing Results for the Pre-Crunch State Early 2007}

We value resetting and non-resetting forward CDO contracts for a range of forward starting dates T. The data used is for the European iTraxx Main Series 6 index from February 22 2007. The index fixed coupons and traded spreads are shown in table 2 and we use a constant risk-free rate of $4.2 \%$ obtained from the Euro swap curve. The tenor of the forward contracts is always five years i.e. $T^{*}-T=5$. The forward dates we use are 0 years i.e. the spot spread and the 1,3 and 5 year forward starting dates. The forward break-even spreads for the non-resetting and resetting forwards are shown in table 6 .
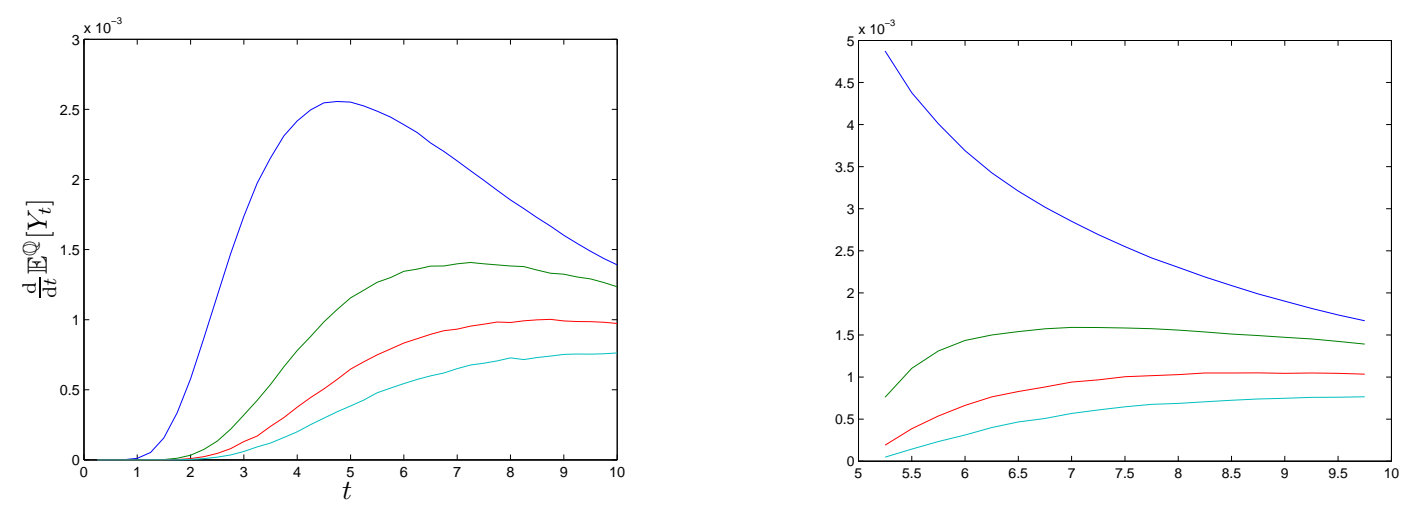

Figure 4: Rate of expected losses in tranches [0,3\%], [3\%,6\%], [6\%,9\%], [9\%,12\%] in 2007, for correlation $\rho=0.5$, for non-resetting (left) and resetting (right) losses.

\subsubsection{Non-Resetting Forward CDO Tranche}

First we focus on the non-resetting tranches. From table 6 we observe the following points:

- As the forward start date increases, the break-even forward spread increases for all tranches.

- For the junior mezzanine (3-6\%) tranche, as the forward start date increases the spread sensitivity to correlation changes sign. The sensitivities of all other tranches are single signed.

Both of these observations can be explained by the fact that losses in the portfolio are cumulative. As time passes, the total loss in the portfolio accumulates and so the attachment and detachment points of non-resetting forward tranches effectively move down the capital structure. In other words, forward equity tranches start behaving like very narrow spot equity tranches, forward 


\begin{tabular}{|c|c|c|c|c|c|c|c|}
\hline \multirow[t]{2}{*}{$\rho=0.1$} & \multicolumn{4}{|c|}{ non-resetting } & \multicolumn{3}{|c|}{ resetting } \\
\hline & $T=0$ & $T=1$ & $T=3$ & $T=5$ & $T=1$ & $T=3$ & $T=5$ \\
\hline $0 \%-3 \%$ & $7.55 \%$ & $24.06 \%$ & $51.32 \%$ & $64.31 \%$ & $24.05 \%$ & $50.26 \%$ & $60.95 \%$ \\
\hline $3 \%-6 \%$ & 15.6 & 71.0 & 374.2 & 911 & 70.9 & 312.3 & 484.0 \\
\hline $6 \%-9 \%$ & 0.7 & 4.7 & 58.7 & 231 & 4.7 & 43.5 & 79.5 \\
\hline $9 \%-12 \%$ & 0 & 0.7 & 9.7 & 54 & 0.7 & 6.6 & 10.3 \\
\hline $12 \%-22 \%$ & 0 & 0 & 0.7 & 4.5 & 0 & 0.3 & 0.5 \\
\hline $22 \%-100 \%$ & 0 & 0 & 0 & 0 & 0 & 0 & 0 \\
\hline \multicolumn{8}{|l|}{$\rho=0.3$} \\
\hline $0 \%-3 \%$ & $2.14 \%$ & $13.87 \%$ & $31.87 \%$ & $36.77 \%$ & $13.87 \%$ & $33.04 \%$ & $42.44 \%$ \\
\hline $3 \%-6 \%$ & 86.4 & 174.2 & 441.9 & 704.3 & 174.1 & 402.9 & 545.3 \\
\hline $6 \%-9 \%$ & 25.0 & 62.6 & 188.5 & 358.6 & 62.6 & 164.1 & 231.1 \\
\hline $9 \%-12 \%$ & 8.2 & 24.1 & 88.1 & 197.6 & 24.1 & 74.4 & 107.9 \\
\hline $12 \%-22 \%$ & 1.7 & 5.0 & 24.3 & 63.2 & 5.0 & 19.9 & 25.8 \\
\hline $22 \%-100 \%$ & 0 & 0.1 & 0.4 & 1.3 & 0 & 0.2 & 0.2 \\
\hline \multicolumn{8}{|l|}{$\rho=0.5$} \\
\hline $0 \%-3 \%$ & $-3.48 \%$ & $5.18 \%$ & $17.96 \%$ & $20.16 \%$ & $5.19 \%$ & $20.11 \%$ & $28.31 \%$ \\
\hline $3 \%-6 \%$ & 116.2 & 193.9 & 400.8 & 532.2 & 193.8 & 384.5 & 507.8 \\
\hline $6 \%-9 \%$ & 54.5 & 101.6 & 228.2 & 341.4 & 101.5 & 211.2 & 283.7 \\
\hline $9 \%-12 \%$ & 28.6 & 59.8 & 142.9 & 237.0 & 59.7 & 128.9 & 177.0 \\
\hline $12 \%-22 \%$ & 9.8 & 22.0 & 62.0 & 118.5 & 22.0 & 54.4 & 73.9 \\
\hline $22 \%-100 \%$ & 0.3 & 0.8 & 2.9 & 6.4 & 0.8 & 2.3 & 2.1 \\
\hline
\end{tabular}

Table 6: The non-resetting and resetting forward spreads (bp) for varying values of the correlation parameter. The equity tranches are quoted as an upfront assuming a 500bp running spread. The model is calibrated to the iTraxx Main Series 6 index for 22 Feb 2007. All forwards have a tenor of 5 years. 
junior mezzanine tranches start behaving like spot equity tranches and so on. On the forward start date, investors will require additional compensation for holding these now riskier tranches and so the break-even forward spread increases.

This also gives the reason why the correlation sensitivity of the junior mezzanine tranche changes sign. For a start date sufficiently far into the future, the tranche is expected to be an equity tranche which has a negative correlation sensitivity.

\subsubsection{Resetting Forward CDO Tranche}

Now we turn to the resetting tranches which from a dynamic modelling point of view can be considered the more interesting of the forward contracts. From table 6 we observe the following points:

- For the forward tranches with a start date in 1 years time the break-even spreads are the same as for the non-resetting forward tranches.

- As the forward start date increases, the break-even forward spread date generally increases.

Addressing these observations in order, the reason for the first point is the nature of the structural model. Because of the diffusive nature of the asset processes, the probability of defaults occurring in the short term is very low. In Section 3 this was highlighted as one of the major downsides for this type of model. The consequence of that property here is that the cumulative loss within the first year is negligible and so we have $\hat{L}_{t} \approx L_{t}$. This in turn leads to the same break-even spreads for both types of forward contract.

The second point can be explained simply by the potential for a decrease in credit quality due to the natural diffusion of the asset processes. This is also present in the non-resetting tranche prices but there the increase in spreads is dominated by the move down the capital structure. An exception is the super senior (22-100\%) tranche for high correlation. We come back to this when discussing the distressed state where this effect is more pronounced.

\subsection{Forward Pricing Results for the Distressed State Late 2008}

We again value resetting and non-resetting forward CDO contracts for a range of forward starting dates $T$. The data used is for the European iTraxx Main Series 6 index from December 52008 . The index fixed coupons and traded spreads are shown in table 4 and we use a constant risk-free rate of $3.3 \%$ obtained from the Euro swap curve. The contract details are as before. Forward break-even spreads for the non-resetting and resetting forwards are shown in tables 7 .

Comparing figures 5 with 4 , the expected loss rate peaks at the short end in the distressed environment of 2008. The effect on the resetting forward starting CDO, which is basically a standard CDO moved into the future, is that the tranche spreads decrease with the forward start date. This is in contrast to the 2007 environment where the risk is generally perceived to increase with the forward start date.

The behaviour is more involved for the non-resetting tranches, where the outstanding tranche notional decays leading up to the forward start date. The net effect here is that typically the 


\begin{tabular}{|c|c|c|c|c|c|c|c|}
\hline \multirow{2}{*}{$\rho=0.3$} & \multicolumn{4}{|c|}{ non-resetting } & \multicolumn{3}{|c|}{ resetting } \\
\hline & $T=0$ & $T=1$ & $T=3$ & $T=5$ & $T=1$ & $T=3$ & $T=5$ \\
\hline $0 \%-3 \%$ & $81.88 \%$ & $82.40 \%$ & $69.26 \%$ & $56.96 \%$ & $83.21 \%$ & $77.69 \%$ & $68.98 \%$ \\
\hline $3 \%-6 \%$ & 2275.2 & 3106.5 & 2658.7 & 1989.3 & 2479.9 & 1830.2 & 1206.6 \\
\hline $6 \%-9 \%$ & 1273.1 & 1708.0 & 1771.4 & 1401.2 & 1348.2 & 901.9 & 522.1 \\
\hline $9 \%-12 \%$ & 775.7 & 1045.9 & 1205.5 & 1024.9 & 811.7 & 487.7 & 238.9 \\
\hline $12 \%-22 \%$ & 307.8 & 425.3 & 570.1 & 546.6 & 313.3 & 143.9 & 51.4 \\
\hline $22 \%-100 \%$ & 9.2 & 13.9 & 24.1 & 29.4 & 8.0 & 1.8 & 0.2 \\
\hline \multicolumn{8}{|l|}{$\rho=0.5$} \\
\hline $0 \%-3 \%$ & $69.56 \%$ & $66.22 \%$ & $47.22 \%$ & $34.18 \%$ & $70.09 \%$ & $63.07 \%$ & $53.86 \%$ \\
\hline $3 \%-6 \%$ & 1743.2 & 2090.3 & 1580.0 & 1119.3 & 1858.6 & 1441.5 & 1033.5 \\
\hline $6 \%-9 \%$ & 1079.7 & 1341.4 & 1149.0 & 869.2 & 1141.9 & 845.4 & 565.4 \\
\hline $9 \%-12 \%$ & 748.6 & 938.6 & 890.4 & 690.9 & 787.1 & 549.8 & 333.5 \\
\hline $12 \%-22 \%$ & 384.7 & 495.5 & 532.2 & 455.6 & 400.0 & 241.7 & 123.6 \\
\hline $22 \%-100 \%$ & 25 & 33.4 & 44.8 & 44.8 & 23.3 & 8.6 & 2.5 \\
\hline \multicolumn{8}{|l|}{$\rho=0.7$} \\
\hline $0 \%-3 \%$ & $56.25 \%$ & $49.41 \%$ & $29.70 \%$ & $16.99 \%$ & $55.67 \%$ & $47.50 \%$ & $38.76 \%$ \\
\hline $3 \%-6 \%$ & 1374.6 & 1481.6 & 1040.3 & 734.7 & 1428.3 & 1146.6 & 864.3 \\
\hline $6 \%-9 \%$ & 931.3 & 1072.1 & 827.8 & 602.8 & 973.7 & 766.2 & 549.2 \\
\hline $9 \%-12 \%$ & 695.8 & 818.8 & 689.6 & 516.9 & 731.3 & 554.3 & 379.7 \\
\hline $12 \%-22 \%$ & 418.1 & 513.8 & 478.1 & 384.8 & 443.0 & 310.4 & 188.1 \\
\hline $22 \%-100 \%$ & 44.5 & 55.9 & 64.1 & 57.9 & 42.7 & 20.8 & 9.5 \\
\hline
\end{tabular}

Table 7: The non-resetting and resetting forward spreads (bp) for varying values of the correlation parameter. The equity tranches are quoted as an upfront assuming a 500bp running spread. The model is calibrated to the iTraxx Main Series 6 index for 5 Dec 2008. All forwards have a tenor of 5 years. 

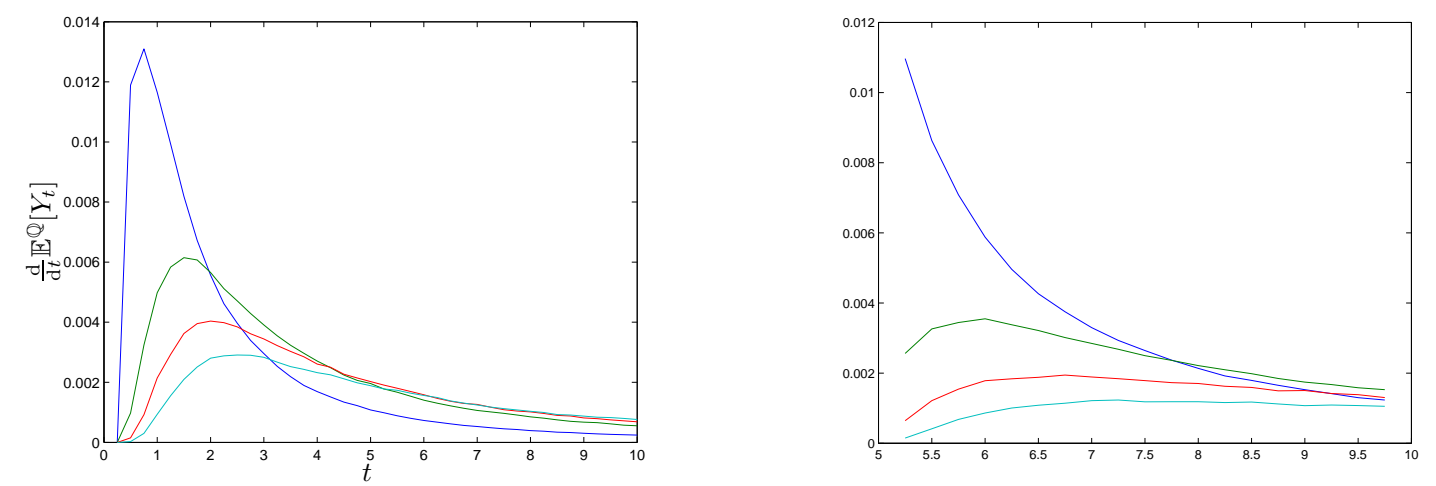

Figure 5: Rate of expected losses in tranches [0,3\%], [3\%,6\%], [6\%,9\%], [9\%,12\%] in 2008, for fixed correlation $\rho=0.5$, for non-resetting (left) and resetting (right) losses.

spreads decrease, with respect to the forward start date, for the junior tranches, increase for the senior tranches, and have a hump-shaped term-structure in the mezzanine range.

\section{Conclusions}

We have illustrated the ability of our simple model to calibrate to the index term-structure in wildly different market environments, and have shown that the correlation sensitivity of tranche spreads demonstrates the behaviour expected. More importantly, using just two parameters and without making them time-dependent, we have shown that our very simple structural evolution model displays realistic term-structure dynamics. Using just the volatility parameter, it is able to calibrate well to all three index spreads and correlation sensitivities of the various tranches are fairly stable across maturities. This is a significant improvement on the majority of pricing models which lack a coherent means of incorporating dynamics. The next stage, which has not been the focus here, is to extend the framework so that it can calibrate to all tranches with a single set of parameters. This will by necessity involve moving away from a simple Brownian Motion driving the process to a more general Levy or jump-diffusion process allowing the loss distribution process to become more skewed, allocating more weight to the tail and increasing super senior tranche spreads.

\section{References}

[1] Bayraktar, E. and Yang, B. Multi-scale Time- Changed Birth Processes for Pricing MultiName Credit Derivatives, to appear in Appl. Math. Finance

[2] Brigo, D. \& Pallavicini, A. \& Torresetti, R. (2006) Calibration of CDO tranches with the dynamical generalized-Poisson loss model. Working paper. 
[3] Black, F. \& Cox, J. (1976) Valuing Corporate Securities: Some Effects of Bond Indenture Provisions, J. Finance, 31, 351-367.

[4] Cariboni, J., \& Schoutens, W. (2004) Pricing Credit Default Swaps Under Lévy Models, UCS report 2004-2007, K.U.Leuven, Leuven.

[5] Carmona, R., Crepéy, S. (2009) Importance sampling and interacting particle systems for the estimation of Markovian credit portfolio loss distributions. Working paper.

[6] Carmona, R., Fouque, J.-P. \& Vestal, D. Interacting particle systems for the computation of rare portfolio losses. Working paper.

[7] Di Graziano, G., \& Rogers, C. (2006) A dynamic approach to the modelling of correlation credit derivatives using Markov chains. Working paper.

[8] Duffie, D., \& Lando, D. (2001) Term Structure of Credit Spreads with Incomplete Accounting Information, Econometrica, 69, 633-664.

[9] Errais, E. \& Geisecke, K. \& Goldberg, L. (2006) Affine point processes and portfolio credit risk. Working paper.

[10] Filipovic, D., Overbeck, L., \& Schmidt, T. (2008) Dynamic CDO Term Structure Modelling, to appear in Math. Finance.

[11] Finger, C., Finkelstein, V., Pan, G., Lardy, J., Thomas, T. \& Tierney, J. (2002) Creditgrades $^{\text {TM }}$. Technical Document, RiskMetrics Group, Inc. Modelling

[12] Fouque, J., Wignall B. \& Zhou, X. (2006) Modeling Correlated Defaults: First Passage Model Under Stochastic Volatility. Working Paper.

[13] Giles, M. \& Carter, R. (2006) Convergence analysis of Crank-Nicolson and Rannacher timemarching, J. Comp. Fin., 9(4), 89-112.

[14] Hambly, B.M. \& Jin, L. (2008) SPDE approximations for large basket portfolio credit modelling, in preparation.

[15] Haworth, H., \& Reisinger, C. (2007) Modelling Basket Credit Default Swaps with Default Contagion, J. Credit Risk, 3(4), 31-67.

[16] Hilberink, B., and Rogers, L. C. G. (2002) Optimal Capital Structure and Endogenous Default, Fin. Stoch., 6, 237-263.

[17] Hull, J., \& White, A. (2001) Valuing Credit Default Swaps II: Modeling Default Correlations, Journal of Derivatives, 8, 12-22.

[18] Hull, J., Predescu M., \& White, A. (2005) The Valuation of Correlation-Dependent Credit Derivatives Using a Structural Model. Working Paper, University of Toronto. 
[19] Hull, J., \& White, A. (2006) Forwards and European Options on CDO Tranches. Working paper.

[20] Jackson, K., \& Zhang, W. (2007) Valuation of Forward Starting CDOs, Working Paper, University of Toronto.

[21] Joshi, M., \& Stacey, A. (2005) Intensity gamma, a new approach to pricing portfolio credit derivatives. Working paper.

[22] Krylov, N.V. (1994) A $W_{2}^{n}$-theory of the Dirichlet problem for SPDEs in general smooth domains, Probab. Theory Relat. Fields, 98, 389-421.

[23] Merton, R. (1974) On the Pricing of Corporate Debt: the Risk Structure of Interest Rates, J. Finance, 29, 449-470.

[24] Mortensen, A. (2006) Semi-analytical valuation of basket credit derivatives in intensity-based models, J. Derivatives, 13(4), 8-26.

[25] O'Kane, D. (2008) Modelling Single-name and Multi-name Credit Derivatives. Wiley Finance.

[26] Papageorgiou, E. and Sircar, R. (2009) Multiscale Intensity Models and Name Grouping for Valuation of Multi-name Credit Derivatives, to appear in Appl. Math. Finance.

[27] Pooley, D. M., Vetzal, K. R., \& Forsyth, P. A. (2003) Remedies for non-smooth payoffs in option pricing, J. Comp. Fin., 6, 25-40.

[28] Rannacher, R. (1984) Finite Element Solution of Diffusion Problems with Irregular Data, Numerische Mathematik, 43, 309-327.

[29] Schönbucher, P. J. (2005) Portfolio losses and the term structure of loss transition rates: a new methodology for the pricing of portfolio credit derivatives. Working Paper.

[30] Sircar, R. and Zaraphopolou, T. (2008) Utility valuation of multiname credit derivatives and applications to CDOs. to appear in Quant. Finance

[31] Tavella, D., \& Randall, C. (2000) Pricing Financial Instruments - The Finite Difference Method. Wiley.

[32] Zhou, C. (1997) A Jump-Diffusion Approach to Modelling Credit Risk and Valuing Defaultable Securities, Federal Reserve Board, Washington.

[33] Zhou, C. (2001a) The Term Structure of Credit Spreads with Jump Risk, J. Bank. Fin, 25, $2015-2040$

[34] Zhou, C. (2001b) An Analysis of Default Correlations and Multiple Defaults, Rev. Fin. Studies, 14, 555-576. 